\title{
Labyrinthe
}

32 | 2009 (1)

Le petit théâtre intellectuel

\section{Daniel Schneidermann, ou Le Renégat universel ?}

\section{Guillaume Paugam}

\section{(2) OpenEdition}

\section{Journals}

Édition électronique

URL : http://journals.openedition.org/labyrinthe/4005

DOI : $10.4000 /$ labyrinthe.4005

ISSN : 1950-6031

Éditeur

Hermann

Édition imprimée

Date de publication : 19 juin 2009

Pagination : 127-131

ISBN : 978-2-7056-6885-3

\section{Référence électronique}

Guillaume Paugam, "Daniel Schneidermann, ou Le Renégat universel ? », Labyrinthe [En ligne], 32 | 2009

(1), mis en ligne le 01 février 2011, consulté le 19 avril 2019. URL : http://journals.openedition.org/

labyrinthe/4005 ; DOI : 10.4000/labyrinthe.4005 


\section{DANIEL SCHNEIDERMANN, OU LE RENÉGAT UNIVERSEL?}

À l'aube des années 90, Daniel Schneidermann n'était encore que le rubricard TV du supplément dominical du Monde où, coulant ses signes heureux dans des jours paisibles, il semblait ne gêner personne. À peine une décennie et deux licenciements plus tard (d'un paquebot Monde qui n'en finit plus de sombrer, d'une télévision publique aux abois), le voici doublement réfugié au sein des colonnes d'un Libé qui crie faillite et d'un fragile site internet dédié à la critique des médias ${ }^{1}$ - ignorant donc obstinément que cette critique n'est pas possible ou qu'il doit la pratiquer mal (à en croire ses détracteurs selon lesquels il n'en fait pas assez ou ses employeurs pour qui il est allé trop loin), qu'elle s'avère aussi peu viable économiquement que professionnellement peu judicieuse, que le Porte-parole n'a pour lui que l'attachement à ses causes et jamais la sympathie de ses co-belligérants.

On pourrait du moins louer sa persévérance ou son esprit de suite. Las, perché sur le promontoire éphémère de la souveraine critique qui, s'il n'était par avance sapé par la versatilité de ses structures (économiques, sociales, politiques), menacerait toujours de s'effondrer sur lui-même, il n'est aucun reproche que l'homme ne semble devoir attirer. D'ailleurs, quitte à ne pas le plaindre de son sort, on pourra même l'en rendre responsable et clamer qu'il l'a bien cherché. De fait, pris dans les feux croisés de la critique de l'information et l'appartenance à la caste de ceux qui la font, Schneidermann ne semble avoir investi le champ de tir que pour y trôner à la place d'honneur: tout à la fois cible auto-désignée et suicidé professionnel, il apparaît tour à tour dans tous les viseurs. Aussi la singularité du cas Schneidermann tient-elle moins aux rancunes qu'il s'attire pour ce qu'il fait qu'aux animosités qu'il charrie quoi qu'il fasse. Théoricien, certes, mais d'un seul concept - l' « Emballement»-qui, aussi simple qu'éprouvé, ne peut lui valoir aucune position de surplomb, l'homme est concurremment un praticien contrarié: tout à la fois pourfendeur de

1. «@rrêt sur images »: www.arretsurimages.net. 
la médiocrité médiatique et incorrigible zélateur de ses capacités propres à s'autoréguler. Un Expert embedded, donc, instamment jugé part des faiblesses inhérentes au système qu'il ne peut totalement dénoncer et dont la conscience d'observateur participant, affinée par l'exploration interne, se manifeste toujours selon le motif de la schize: partagée entre l'acuité inquisitrice et l'indulgence coupable. Juge pour ses pairs et partie pour ses adversaires, au final l'homme se retrouve toujours suspect. Quand il dénonce la propension de ses confrères à s'appesantir sur les moindres faits et gestes de l' " hyper-Président ", ne participe-t-il pas lui-même à la polarisation de l'information qu'il semble dénoncer? S'épanche-t-il sur telle collusion des médias avec les pouvoirs? C'est qu'il savait, qu'il pouvait voir, qu'il devait révéler! Quant au silence obséquieux des médias qu'il relève sur telle affaire récente, n'avait-il vraiment à sa disposition aucun levier pour le briser? Membre du clan, il est forcément part de l'omerta... Les dents de ses confrères grincent, sa hiérarchie hoquette, les contempteurs des médias s'étranglent. Question de support également. Éditorialiste récidiviste devenu Blogueur impénitent ${ }^{1}$, sa conversion, somme toute tardive, aux vertus du web n'est-elle pas fourbe qui tente d'y calquer le modèle obsolète d'une presse payante? Piteuse Pythie, Momie qui se voudrait Cassandre... Serait-elle trop aveugle pour percevoir ses propres égarements? Arrivée trop tard pour les uns et trop avant-gardiste pour les siens, se figurant qu'au devant il n'était personne, elle se retourna pour s'apercevoir qu'elle était seule...

Au départ, un pari : la critique des médias pratiquée au sein des médias eux-mêmes. Intention louable; sauf qu'en fait de louange, notre homme se vit sitôt désigné à la vindicte générale - pour des motifs, d'ailleurs, pas toujours compatibles et que seule une fortune remarquable dans la collection des animosités lui aura permis d'agréger. D'où cette suite de carrière en dents de scie et la subséquente litanie d'inimitiés auprès de laquelle Les Confessions de Jean-Jacques font figure de long poème galant (la lapidation de Motiers comprise). S'attirant les foudres de ses pairs pour son intransigeance (quand ses opposants fustigent ses compromis), Schneidermann fut tour à tour vanté pour sa ténacité et sa

\footnotetext{
1. Sur le « Big Bang Blog» d'abord (www.bigbangblog.net) où l'Internet devait faire vaciller les médias traditionnels, désormais avec un billet quotidien, le «neuf-quinze », proposé parmi les contenus gratuits du site «@rrêt sur images» (aussi appelé chronique des « matinautes », voir: http://www. arretsurimages.net/chroniqueur.php?id=12).
} 
rigueur professionnelle puis dénoncé pour son obstination et son caractère psychorigide. Parfois l'inverse. Parfois en même temps. Plus rarement par les mêmes (comprendre: les stratèges de sa direction, au moment de le lâcher). Pour les uns, l'énoncé des griefs ne tient qu'à son programme censé contenir en germe sa propre contradiction: il ne saurait y avoir de critique pertinente du système à l'intérieur du système. Ce lui valut du moins une mémorable empoignade avec Pierre Bourdieu dont, postmortem, les thuriféraires nourrissent encore la flamme avec dévotion, gardant toujours un œil braqué sur l'animateur honni du maître.

Ainsi la Télévision, se plaisait à penser le sociologue, ne saurait assurer sa propre critique (sinon sous une forme anodine qui ne risque pas de lui nuire). Provocateur, le journaliste l'invita à étayer cette opinion in praesentia sur son plateau. L'émission se passa mal, preuve que Bourdieu avait raison (et non, évidemment, qu'il avait été mauvais). D'analyses de l'un en réponses de l'autre, plusieurs colonnes de journaux ${ }^{1}$ et deux livres plus $\operatorname{tard}^{2}$, la discussion tourna au combat de coq; il faut dire que la constance est, pour qui fait profession de parler en public, une vertu. Chacun, donc, se cantonna sagement à son rôle: le sociologue à l'investigation du système médiatique, l'animateur à l'analyse des informations produites. D'où une seconde série d'antipathies, internes à son champ cette fois. À l'heure où la dénonciation d'une erreur journalistique passe pour une atteinte aux libertés ${ }^{3}$ (de la presse, de l'expression), l'entreprise de Schneidermann avait peu de chances de s'attirer les bonnes grâces de ses pairs, moins encore de provoquer leur sympathie. Extérieur au métier, il fût passé pour un vil dénonciateur - ingrat, injuste, ignorant des contraintes propres de l'exercice et donc candidat tout désigné au dédain du sérail, ses oukases auraient été déclarés d'autant moins pertinents qu'ils étaient lointains. Rien d'autre qu'un Pamphilet vociférant auquel il vaut mieux ne pas prêter l'oreille (il la rendrait toute abîmée). Enfant de la balle, c'est un traître, un faux-frère, un Renégat. D'ailleurs, de la

\footnotetext{
1. http://www.monde-diplomatique.fr/1996/04/BOURDIEU/2633; http://www.monde-diplomatique. fr/1996/05/SCHNEIDERMANN/2778.html [consultés le 15/02/2009].

2. Pierre Bourdieu, Sur la télévision, Raisons d'agir, Paris, 1996; Daniel Schneidermann, Du journalisme après Bourdieu, Fayard, Paris, 1999.

3. D'une journaliste du Monde à son confrère de Libération: «Bonjour Nicolas, je vous ai connu plus confraternel, c'est vraiment sympa d'être taxée de faire du mauvais journalisme, surtout en cette période sombre pour la presse écrite et la liberté d'expression ». http://cordonsbourse.blogs.liberation. fr/cori/2009/01/parachutes-dors.html\#comment-145002618 [consulté le 15/02/2009].
} 
balle, l'est-il vraiment? Le cirque médiatique lui dénie sans vergogne ses titres de noblesse. L'a-t-on vu risquer sa peau à Gaza, en Afghanistan, sur tel « théâtre »? La critique est facile, Môssieur le journaliste de bureau ${ }^{1}$, mais l'art de l'article bien troussé, du montage adéquat, du traitement du sujet... Attaquée, la tribu arbore aussitôt ses mythes: " N'est pas Albert Londres qui veut! » (le totem Albert Londres dût-il cacher bien des charniers de Timisoara).

Ces feux croisés finirent par avoir raison du programme - cette émission pas assez vivante, son format vieillot, sa formule dépassée. Son indécente liberté de ton. La télévision doit divertir, sa critique s'avère si ennuyeuse. Il convenait de la déprogrammer - preuve que Bourdieu avait raison (mais non raison de croire qu'effectivement l'émission dérangeait). Pourtant Schneidermann aura beau être le premier réfugié internet du sarkozysme décomplexé des médias, cela n'empêchera pas ses détracteurs alter de s'acharner, le poursuivant jusque sur la toile où il comptait se refaire une virginité. Dans une enquête participative qu'il estimerait sans doute louable si elle ne l'avait pris pour objet, les internautes rappellent ses hauts-faits: soupçon de plagiat, compromis de couloirs, licenciement abusif, etc. Autant de pierres dans son jardin. Que sa notice Wikipédia s'enrichisse ${ }^{2}$, c'est son passif qui s'alourdit d'autant. Victime d'un pouvoir de droite, il voit se briser sa façade de gauche: un traître à la cause, que ce soit dit. Son site internet de surcroît, est une gageure (1. Capitaliste; 2. Antédiluvienne; 3. Utopiquement Benoîte; rayer la mention inutile). Un abonnement? À l'heure du participatif et du tout publicitaire? L'internaute lambda rechigne, et il n'est jusqu'aux morandiniens invétérés (adeptes de cet autre ennemi héréditaire et concurrent autodéclaré de Schneidermann) pour lui faire la leçon sur les faiblesses flagrantes de son modèle économique ${ }^{3}$. Il est vrai qu'il vaut mieux compter sur les libéralités désintéressées du papivore et marchand d'armes Lagardère.

Quelles que soient ces récriminations dont il est le seul dénominateur commun, qu'elles émanent des bourdieusiens inconditionnels ou des

\footnotetext{
1. Comme on désigne par le sobriquet d'armchair anthropologists la tradition des ethnologues en chambre.

2. http://www.bigbangblog.net/article.php3?id_article $=298$ [consulté le 15/02/2009].

3. http://jeanmarcmorandini.tele7.fr/article-23093-arret-sur-image-la-chaine-de-schneidermann-faitun-bide.html [consulté le 15/02/2009].
} 
consciencieux professionnels des médias, à la télévision, sur papier ou sur internet, Schneidermann énerve, ennuie ou inquiète. Figure paternaliste et sentencieuse du juge quand il gourmande l'honnête citoyen et le rappelle à la loi - mais dont ici la légitimité est toujours critiquable, la probité à vérifier et les sentences toujours en question - Schneidermann a la laideur de notre mauvaise conscience: haïssable quand elle dit vrai, vouée aux gémonies quand elle a l'heur de se fourvoyer. Mouton galeux et bouc émissaire de sa profession, l'homme semble voué à assumer son étrange destin de figure expiatoire au carrefour de nos grégarités. 